\title{
Sony Labou Tansi, afflux des écrits et flux de l'écriture
}

\author{
Nicolas Martin-Granel
}

\section{OpenEdition}

Journals

Édition électronique

URL : https://journals.openedition.org/coma/260

DOI : $10.4000 /$ coma.260

ISSN : 2275-1742

\section{Éditeur}

Institut des textes \& manuscrits modernes (ITEM)

\section{Référence électronique}

Nicolas Martin-Granel, « Sony Labou Tansi, afflux des écrits et flux de l'écriture », Continents manuscrits [En ligne], 1 | 2014, mis en ligne le 22 avril 2014, consulté le 18 juin 2021. URL : http:// journals.openedition.org/coma/260; DOI : https://doi.org/10.4000/coma.260

Ce document a été généré automatiquement le 18 juin 2021.

\section{(C) $\odot \Theta \Theta$}

Continents manuscrits - Génétique des textes littéraires - Afrique, Caraîbe, dispora est mis à disposition selon les termes de la licence Creative Commons Attribution - Pas d'Utilisation

Commerciale - Pas de Modification 4.0 International. 


\title{
Sony Labou Tansi, afflux des écrits et flux de l'écriture
}

\author{
Nicolas Martin-Granel
}
Je
boxe titre d'homme
en
jeu -
J'éparpille
mon
droit
de cité
dans l'orage
des cellules -
Je m'insurge à croire
qu'il était une fois -
Je désorganise
la genèse $e^{1}$.

1 "La Vie et Demie, ça s'appelle écrire par étourderie » : tel est l'avertissement qu'un jeune écrivain congolais lançait en 1979 à ses lecteurs au seuil de son premier roman publié au Seuil. Ça commence bien, Sony expliquant son « étourderie » comme une allusion au vol du premier cahier manuscrit de son roman dans le train pour Pointe-Noire :

Alors j'ai commencé à écrire La Vie et demie. Quand j'ai fini d'écrire, c'est-à-dire mars, avril, mai, juin, juillet. En août je voulais aller à Pointe-Noire pour passer des vacances. J'avais le cahier, puisque j'écris toujours sur des gros cahiers. J'avais mon cahier dans ma mallette. J'ai pris le train et on avait volé la mallette. J'ai perdu la première version de La Vie et demie. Elle doit traîner quelque part, sinon peut-être qu'elle est toute bouffée par je sais pas quoi. Je suis allé à Pointe-Noire pour me reposer, je ne me suis pas reposé. Je me suis mis à réécrire en essayant de me rappeler. Si vous voyez bien dans La Vie et demie il y a une sorte de musicalité qui revient pour essayer de retrouver quelque chose. Il y a des moments comme ça dans la Vie et demie, parce que j'étais obligé de me rappeler les chapitres, de me rappeler... C'était difficile, c'était douloureux, mais je devais le faire. Je l'ai fait à 
moitié, j'ai fait sûrement une chose différente, mais il y a eu ces circonstances dans lesquelles ce livre a été écrit. Et puis quand il a été fini, alors ça s'explique aussi de parler d'étourderie en ce moment-là, parce que j'ai perdu peut-être mon manuscrit par étourderie aussi. En fait, ça se comprend, moi je le comprends comme ça. Vous, vous le comprenez comme vous voulez le comprendre.

2 Pour un chercheur qui tente à présent de dresser un état des lieux, avec méthode et conformément aux normes académiques de la génétique textuelle, il nous faut bien l'avouer au seuil de cet essai, les manuscrits de Sony Labou Tansi (SLT), traces concrètes de son écriture qui « boxe », ont de quoi provoquer quelque étourdissement.

3 S'étourdir, en rester étourdi comme un boxeur, c'est en effet le risque délibéré qu'il faut prendre pour entrer dans le "labyrinthe" discontinu et chaotique des écrits signés SLT (mais parfois d'hétéronymes instables), et pour tenter de poursuivre le fil continu de son écriture.

\section{Écrits éparpillés : «Ces cadavres qui aspirent à la résurrection »}

Quelques mois après la mort de SLT survenue le 14 juin 1995, alors même que nous appréhendions la disparition totale de ses archives, un premier travail systématique de localisation et d'inventaire des manuscrits et des livres se trouvant dans la chambrebureau de l'auteur a pu être mené à l'initiative de l'ANEC (Association Nationale des Écrivains au (ongo ${ }^{2}$ ) et à l'occasion de la commande d'un livre ${ }^{3} \mathrm{~d}^{\prime}$ hommage par la mission de Coopération et d'Action Culturelle au Congo. Réalisé au cours de l'année 1996 par des proches de SLT restés sur place, notamment Victor Mbila Mpassi (son cousin et tuteur de ses trois filles alors mineures), Apollinaire Singou-Basseha ${ }^{4}$ (journaliste) et moi-même, ce premier travail exploratoire du fonds personnel de l'auteur a permis d'inventorier, classer et numéroter toutes les archives, manuscrites et tapuscrites, sous forme de cahier ou de feuillets volants, qui avaient été abandonnées dans le plus grand désordre au bas de sa petite bibliothèque vitrée, dans un état de dégradation assez avancé. La première opération de sauvetage a consisté à mettre ces documents, souvent endommagés du fait de l'humidité ambiante et des divers insectes, à l'abri dans une cantine déposée dans la maison de Sony, et la seconde à en photocopier toutes les pages une à une. Ce travail était presque achevé lorsque survint la guerre civile de juin 1997. Rapatrié manu militari, j'ai pu heureusement sauver du pillage général, en les emportant dans mon seul baluchon autorisé, l'essentiel des cahiers photocopiés et aussi les quelques originaux qui étaient déposés chez moi en attente de copie. Par la suite, nous avons reçu des informations contradictoires sur le sort de la cantine renfermant les manuscrits qui aurait disparu lors des terribles "événements » de $1998^{5}$ qui se sont déroulés dans les quartiers sud (Bacongo et Makélékélé où se trouvait la maison de Sony). Il fallait en avoir le cœur net; un nouvel état des lieux s'imposait. L'occasion allait bientôt se présenter - en poste au Cameroun à partir de 1999, je pouvais rejoindre facilement le Congo voisin -, ainsi qu'un renfort précieux en la personne de Greta Rodriguez Antoniotti, jeune doctorante travaillant sur le théâtre de Sony et désireuse de documenter sa recherche sur le vif du terrain congolais.

5 Lors de notre séjour à Brazzaville au mois d'avril 2003, nous avons pu enfin vérifier sur place l'étendue des dégâts. En fait, ce fut une bonne surprise de constater que si la 
cantine avait en effet disparu, son contenu non ! Il était seulement rejeté et entassé en vrac dans le bas de la même bibliothèque. Retour à la case départ? Pas tout à fait, puisque, après un rapide inventaire des manuscrits survivants, ceux-ci comptaient, en plus de ceux déjà répertoriés en 1996, au moins deux nouveaux cahiers inconnus, lesquels avaient surgi, comme par magie ou génération spontanée, d'on ne sait quelles oubliettes. Ils avaient pour titre : Déjà...j'ai habité tous ces mots (sous la signature de Sony La Boutansi) et Ces hommes qui fatiguent les chiffres (signé SONY Lab'ou Tansi). La quarantaine de pages de ce dernier a fait l'objet d'un enregistrement complet sur photos numériques, et ce grâce aux soins acharnés et minutieux de Greta Rodriguez assistée de Bill Kouélany ${ }^{6}$ qui tenait la bougie ! Malgré l'heure tardive et les contraintes de stockage de l'appareil, elles ont pu ramener du trou noir presque une centaine de clichés de ces manuscrits perdus et (re)trouvés. Parmi eux, le dernier découvert, Ces hommes..., constitue sans doute une trouvaille capitale pour la constitution de l'avanttexte de L'Anté-Peuple, roman qui s'est d'abord appelé La Natte ; le chaînon manquant dans l'évolution du chantier qui mène des ébauches de jeunesse à La Natte : ce titre s'y trouve en effet comme titre de chapitre.

6 Entre-temps, lors de ma résidence professionnelle au Cameroun, le texte de la plupart des cahiers disponibles (la prose romanesque) a été déchiffré et saisi page à page ${ }^{7}$ selon le procédé le plus fidèle à l'original manuscrit et le plus pertinent pour l'étude et l'édition génétique. Soit près de huit cents pages de transcription diplomatique qui restent à exploiter, étudier et publier à l'usage des chercheurs en génétique textuelle. Cependant, c'est à un plus large public que devaient s'adresser les textes inédits destinés à commémorer le dixième anniversaire de la mort de SLT en 2005. Dans le premier projet éditorial élaboré en 2003 par Gréta et moi-même ${ }^{8}$, nous avions opté pour la simple transcription linéarisée de trois ébauches romanesques de jeunesse constitutives du dossier génétique de L'Anté-Peuple. Pour le second projet - le précédent ayant échoué à retenir l'intérêt d'aucun éditeur francophone -, nous décidons de livrer au lectorat amateur de Sony des textes inédits, certes sans notes ni variantes, mais sans qu'aucune retouche (excepté orthographique) soit apportée au tapuscrit ou au manuscrit original, ainsi que nous l'annonçons dans le prospectus éditorial :

L'Atelier de Sony Labou Tansi, ou comment être attentif aux angles ignorés de la création, comment rendre sensibles et le souffle et le travail de l'écrivain à l'œuvre : aux prises avec ses manuscrits. Car si « le manuscrit est l'atelier» (H. Meschonnic), il convenait de ne pas le perdre de vue, de le donner à voir par quelques fac-similés qui sont autant de fenêtres sur le manuscrit: matière première, encore incandescente, du livre à venir. Un atelier, donc, en trois volumes: les lettres donnent accès aux « affres » de l'écriture au quotidien; les «poèmes » au mélange des genres et à la diversité des versions; le «roman» à la version inédite d'une "prose qui boxe».

On ne voit que la part de mon écriture qui crie. On ne voit pas celle qui écrit, disait Sony, pour le regretter sans doute. Pour cette édition, n'a été apportée aucune « aide »arbitrairement? - savante aux textes, et toute norme éditoriale susceptible de «canaliser» l'écriture éruptive de l'écrivain a été congédiée. Sans prétendre proposer une édition génétique, les éditeurs ont tenté de livrer les textes au plus près des manuscrits et tapuscrits originaux.

7 Et c'est bien ainsi ${ }^{9}$ que nous avons établi le texte des trois volumes L'Atelier de Sony Labou Tansi, « coffret anniversaire » qui sera publié en effet en juin 2005, exactement dix ans après la disparition de SLT. Cette parution coïncide aussi avec les «Francofffonies », nom de l'événement saluant l'invitation et la reconnaissance des écrivains de la francophonie par le Salon du Livre de 2006. À cette occasion, le directeur 
de l'Institut des Textes et Manuscrits modernes (ITEM), Pierre-Marc de Biasi, qui a noté l'importance du coffret SLT comme signe précurseur d'un tournant génétique au Sud, s'entretient avec Greta Rodriguez dans la livraison du Magazine Littéraire consacré à l'événement « francofffone ».

La même année, P.M de Biasi dirige un mémoire de Master, «Études génétique de L'Acte de respirer de Sony Labou Tansi ", qui s'appuie sur les manuscrits que G. Rodriguez a mis à la disposition de l'étudiante ${ }^{10}$. Cette collaboration allait bientôt s'organiser de façon systématique et permanente par la création en 2007, au sein même de l'ITEM, d'une équipe «Manuscrit Francophone » et, à l'intérieur de celle-ci, d'un petit groupe Congo composé à l'origine de Daniel Delas et de moi-même. Désormais adoubé de l'autorité scientifique de l'ITEM et du partenariat international de l'Agence Universitaire de la Francophonie, le groupe Congo peut envisager de monter une mission sur le terrain destinée à dresser un nouvel état des lieux du fonds SLT. Cette mission se concrétise en novembre 2008 dans le prolongement d'un colloque consacré à Tchicaya U Tam'si. En voici les résultats ${ }^{11}$ tels qu'ils ont été résumés dans le rapport de mission :

Le reste du séjour a été occupé par des activités concernant Sony Labou Tansi. Il s'est agi pour l'essentiel de sécuriser les manuscrits de l'écrivain, entreposés dans de très mauvaises conditions dans la maison qu'il habitait à Makélékélé, désormais louée à des particuliers. Avec l'accord de la propriétaire, sa fille Darmalla, nous sommes allés dans cette maison, avons distingué manuscrits et livres d'un intérêt secondaire (cahiers d'écolier de ses enfants ou simples livres de la bibliothèque de l'écrivain), fait un inventaire sommaire des manuscrits, transportés ceux-ci dans la maison plus moderne et plus saine de sa fille et mis ceux-ci dans des cantines métalliques (que nous avons achetées), en les munissant d'un numéro d'inventaire et en les photographiant partiellement. Nous avons remis de petites sommes d'argent pour l'achat de cadenas, de produits contre les insectes. D'autres solutions de dépôt ont été envisagées, mais il n'existe pas au Congo de lieux officiels sûrs et les bonnes dispositions de la famille nous ont paru offrir les meilleures garanties. Le reste des livres et le meuble bibliothèque lui-même devraient être transférés bientôt.

Il n'est pas sûr qu'une numérisation « scientifique " puisse être faite dans un avenir proche: d'une part, il n'existe pas au Congo de structures techniques qui le permettent aisément, d'autre part il est peu probable que la sortie des manuscrits, même provisoire et assortie de garanties, soit envisageable. Il faudrait donc étudier la possibilité de coupler la mission de formation envisagée plus haut dans le cadre universitaire avec une mission plus «technique " de conservation et numérisation des manuscrits de Sony Labou Tansi disponibles à Brazzaville, aussi bien ceux déposés chez sa fille Darmalla que ceux détenus par des particuliers comme Nicolas Bissi, dramaturge ami de l'auteur et à présent animateur culturel au CCF, avec qui nous avons repris contact.

Pour le premier fonds, conservé par les ayants droit, l'inventaire que nous avons dressé en urgence la veille de notre départ a permis d'établir un bilan globalement positif par rapport au premier inventaire (voir ELA $\left.n^{\circ} 15,2003\right)$ : sur un total de 42 cahiers manuscrits identifiés, 28 "anciens" retrouvés et 14 «nouveaux » découverts dont 3 ont été entièrement photonumérisés ; 4 « anciens » sont portés manquants : Une vie en arbre... ( $\left.\mathrm{n}^{\circ} 18\right)$, Le Pays intérieur $\left(\mathrm{n}^{\circ} 19\right)$, La Vie et demie $\left(\mathrm{n}^{\circ} 21\right)$, Riposter à sa Gueule ( $\left.\mathrm{n}^{\circ} 22\right)$. Par manque de temps, il n'a pas été possible de faire un point précis sur la présence et l'état des feuillets manuscrits (B) et tapuscrits ${ }^{12}$ (C) regroupés dans une chemise dont on a juste pu noter l'apparence très dégradée par l'infestation d'insectes papivores ${ }^{13}$.

Cependant, cette sauvegarde d'urgence ne pouvait être que qu'une étape provisoire. Le fonds SLT à Brazzaville restait vulnérable du fait de sa matérialité même, soumis aux 
aléas climatiques et politiques qui risquaient de l'endommager, voire de faire disparaître à tout moment. Il était nécessaire d'en garder une copie immatérielle.

Ce qui aboutit lors d'une mission spécifique de numérisation que j'ai pu enfin réaliser, avec le soutien de l'ITEM, durant un mois à Brazzaville, du 14 janvier au 14 février 2011. Au bout du compte, tous les cahiers, au nombre de 43, ainsi que tous les feuillets épars, manuscrits et tapuscrits, littéraires ou non, ont été passés au scanner et enregistrés au format JPEG, soit plus de $4000^{14}$ images d'assez bonne résolution pour être aisément lisibles ${ }^{15}$. Tous les fichiers ont été copiés et mis à disposition à Brazzaville sur l'ordinateur de Victor Mbila Mpassi à l'Institut Français, puis, à mon retour, déposés sur celui du bureau de l'équipe "Manuscrit Francophone » sur le site de l'ITEM de la rue Pouchet.

10 En dehors de ce fonds qui appartient aux ayants droit, il y a nombre de manuscrits (cahiers et lettres) qui se trouvent détenus par les divers amis de Sony. À Brazzaville, Nicolas Bissi, co-fondateur du Rocadu Zulu Théâtre, détient deux ou trois cahiers ${ }^{16}$, qu'il avait eu l'obligeance avant 1997 de me laisser photocopier et qui sont en (petite) partie publiés ${ }^{17}$, mais que je n'ai pu numériser en 2011. En France, les correspondances à José Pivin (voir son fils Jean-Loup P., Revue Noire), à Françoise Ligier (voir sa nièce Ayoko Mensah, Africultures) ont été publiées (dans le coffret SLT), mais restent à numériser. Caya Makhélé détient une version du recueil de poèmes, Le Quatrième Côté du Triangle, dont il annonçait la publication il y a déjà plus dix ans aux éditions Acoria. Voir aussi les fonds de Jean-Michel Devésa, de Guy Lenoir (tous deux à Bordeaux), de Bernard Magnier... dont l'inventaire reste à publier et le contenu à sauvegarder, avec l'accord des ayants droit.

11 En Italie, à Turin, Sergio Zoppi et Antonella Emina m'ont promis de m'envoyer les scans de leur version manuscrite de ce même recueil, qu'ils ont publié ; dans ce même centre de Turin, se trouve aussi un petit fonds Sony-Brésil (correspondance et entretien de Sony avec une universitaire brésilienne)

12 À la bibliothèque municipale de Limoges, dite aussi " pôle francophone » de la BNF, il y a un fonds SLT: Monique Blin, ancienne directrice du Festival, y a déposé de la correspondance et des archives, et RFI ses archives du concours théâtral interafricain ${ }^{18}$.

13 Au Cameroun, le nouveau directeur des éditions CLE, Marcelin Vounda Itoua, que je connaissais bien à Yaoundé comme dirigeant la revue culturelle Patrimoine, avait annoncé il y a quelques années avoir trouvé un manuscrit inédit de SLT (avec d'autres, dont Amadou Hampâté Bâ), qui a pour titre Ici commence ici. Il m'avait fait passer les premières pages, alléchantes en effet. Une mission d'inventaire et de sauvegarde est sans doute à monter dans le fonds de cette maison d'édition qui a édité Conscience de tracteur $^{19}$, la première pièce de théâtre du dénommé « SONY Lab'Ou Tansi ».

\section{Écriture à processus prophétique : «Chair-mots de passe »}

14 Si Sony Labou Tansi s'est assez souvent exprimé sur cette "énergie noire ${ }^{20}$ » qui le poussait à écrire continûment, aussi bien sur le pourquoi («le monde n'avance pas») que le pour/contre quoi (« remettre à plus tard la mort du roman $\left.{ }^{21} »\right)$, il a été bien plus discret sur le comment de son écriture ${ }^{22}$ à jet continu, conçue comme un «acte de respirer ». Je voudrais aborder cette troisième dimension, non plus ce contre quoi (la 
honte du progrès), ni ce sur quoi (la peur du temps) son œuvre a pu avancer, mais comment elle s'avance et s'élance concrètement, à la conquête de l'espace vierge de la page - s'il est vrai qu'il écrit «parce que la page blanche me chagrine et me donne la nausée ».

Pour rendre compte de la progression de l'œuvre, il nous faut peut-être repartir de À quand la vie ?, dernière ébauche de roman, que nous a laissée Sony. Car ce dernier texte est aussi le premier véritable brouillon de l'écrivain. Un brouillon qu'il a relu, corrigé et abondamment raturé. En cela, ce texte est une merveilleuse exception qui nous informe sur la règle ordinaire, ce qu'il appelle la « discipline » en quoi écrire consiste. Tous les autres manuscrits, en effet, donnent l'impression d'avoir été écrits d'un seul jet de plume, sans ébauche préparatoire, sans rature et sans repentir, sans date non plus. Si ce n'est dans À quand la vie ?, Sony ne revient pas - ou si peu - sur le déjà-écrit, il va toujours de l'avant, accumulant les versions qui se suivent sans forcément se ressembler à la lettre. Jour après jour, il reprend son texte pour lui ajouter des pages, presque jamais pour en supprimer. L'œuvre se développe en se multipliant, en se disséminant dans les versions ultérieures. Sa puissance est telle qu'elle explore et épuise tous les possibles: "J'essaie d'avoir un regard explosif et qui va dans tous les sens. "

Pour autant, son créationnisme littéraire n'implique pas qu'il doive ou puisse y avoir création ex nihilo. Il n'y a pas d'origine absolue. Au départ, il y a déjà des mots - un titre souvent, ou bien des germes d'histoire et de scandale, des images brutes qui demandent à devenir verbe et à s'étaler sur des cahiers. Ces images « que nous recevons de la vie, je m'en sers pour écrire des romans. "

17 Ce passage, du chaos aux signes qui lui donnent sens, suppose donc que quelque chose a déjà commencé avant ; et cette chose encore innommée, peut-être innommable, il s'agit seulement de la pousser devant, sur la page encore vierge. L'écriture convertit le temps du déjà-là en espace à parcourir et à découvrir.

Ce qui est une manière de reformuler la question des influences. Que ce soit la vie en général, ou la grand-mère défrichant lentement, plante à plante, son champ, le modèle n'est jamais directement une écriture. Ce n'est même pas une parole. Il est vrai que le parler quotidien a pu inspirer Sony, lui fournir des impressions et des émotions, mais non des expressions à transcrire littéralement. Car pour lui, maitriser une langue revient à en changer quand on passe à l'acte d'écrire - loin de la créolisation à la mode et de tous les métissages faciles. Bien plus, Sony déclare ne pas aimer parler car «la parole est dangereuse, elle nous dément souvent. » De la parole à l'écrire, il y a solution de continuité.

L'écriture est d'un ordre radicalement autre. Elle advient à partir d'un saut qualitatif dans l'espace des lettres, des mots et des pages. C'est ainsi que nous lisons cette notation inscrite sur un agenda :

Un jour, il y en aura qui diront : je l'ai influencé - ils seront nombreux. Et voici ma réponse à tous ceux-là qui croiront qu'ils m'ont influencé = je dirai : d'accord, vous m'avez influencé, mais je suis allé plus loin que vous, j'ai sauté plus haut que vous, accusez moi de cela, pas d'autre chose-, autrement soyez fiers de m'avoir engendré = c'est votre droit après tout. » (5-4-79).

Sony ne dénie pas la dette, il s'en dédie en se dégageant de la continuité historique et génétique par un saut dans l'inédit, que ce soit, comme ici, dans les hauteurs du sublime ou dans les profondeurs de l'abjection. Cette direction est exploitée dans la 
dernière page de La Planète des signes ${ }^{23}$. Ici, au fond de leur cachot, les deux prisonniers ne sauraient esquisser le moindre saut pour échapper à leur condition. La seule issue qui leur reste ouverte, c'est de s'enfoncer davantage, d'aller encore plus bas. C'est pourquoi l'enterré vivant finit par dire :

Il faut creuser. Parce que la vie, c'est là-dedans, on la creuse.

- J'ai lu ça quelque part.

- Life is not a book. Creuse.

Sauter ou creuser, c'est toujours s'évader, se soustraire aux influences du temps, c'est devenir un homme sans influences. Si la vraie vie est ailleurs, elle est certainement dans un espace littéraire ouvert à tous les sauts, à tous les creusements possibles. Avant, c'est plein, trop plein ; devant, au contraire, c'est le vide, un monde qui n'attend que les signes pour être mis au monde.

21 Lire, en revanche, c'est être, de tout façon, sous influence. On aura remarqué que l'injonction finale et péremptoire "creuse" que le personnage adresse à son compagnon de cellule est précédée d'une curieuse maxime énoncée en anglais : «life is not a book. » On aurait tort, me semble-t-il, de la traduire simplement par « la vie n'est pas un roman ", car ce qui est en question ici, ce n'est pas tant le roman comme fiction que comme livre, c'est-à-dire un objet fini et offert à la consommation des lecteurs. Or un lecteur a réponse à tout ; c'est quelqu'un dont le réflexe irréfléchi est de dire : «j'ai déjà lu ça quelque part. » Lire se conjugue au passé de l'accompli, dans le temps du déjà. La lecture participe, pour Sony et selon ses propres mots, de « la fonction honteuse de consommation »; elle réduit le livre à n'être qu'un "comprimé de Nivaquine». De l'autre côté, depuis « l'autre rive ", l'écriture voit le mot d'un autre œil : « un cadavre qui aspire à la résurrection. » L'écrivain est donc un homme qui ne cesse de creuser pour exhumer ces mots cadavres d'où la lecture les a ensevelis. C'est dans cette activité qu'il se sent respirer.

Sony a certes beaucoup lu, mais ses lectures affleurent peu dans ses textes. Ses citations sont rarissimes. On rencontre une seule fois le nom de Verlaine dans ses poésies de jeunesse. Quant aux citations d'auteurs proprement dites, on n'en saurait relever que deux : une dans un entretien et une autre dans un roman. Et elles sont toutes deux d'Antonin Artaud. Le fait est suffisamment remarquable dans la poétique de Sony pour qu'on ne passe pas sur ces citations sans les citer à notre tour. Voici la première qui vient justement souligner le côté aventure de l'écriture : "Vous me permettez de citer Artaud. Il dit: "Nous ne sommes pas encore au monde, les choses ne sont pas encore faites, la raison de vivre n'est pas encore trouvée ». »C'était dans une interview de 1985 à propos des Sept solitudes de Lorsa Lopez. La seconde est sans doute à peu près contemporaine puisqu'elle apparait dans une ébauche de ce même roman, intitulé alors Le Quatrième côté du triangle. Elle aussi situe le travail des mots aux confins de la folie, du hors-sens et du hors-monde :

Pour bien accepter la création il faut ouvrir la porte à la folie, parce que la folie a le pouvoir même d'étrangler les mots pour dire le reste de la réalité en cris, en soupirs, en pieds de son, parce que le mot est un infirme qui ne dit que la réalité expérimentale de l'homme. Le mot, comme dit Artaud, n'est qu'un pèse-nerfs.

Toute la poétique de Sony, inscrite ici de façon tout à fait exceptionnelle dans une fiction, repose sur le postulat que si l'on en reste aux mots de la lecture, la réalité devient rien d'autre que du plagiat, que forme d'agonie organique. 
Si d'aventure le poète en vient à écrire des romans, c'est pour piéger ses lecteurs dans une réalité plagiée. L'État honteux est la plus belle réussite dans ce genre. Sony a souvent avoué sa préférence pour ce roman (parce) que ses lecteurs n'aimaient guère :

Mon désir était d'écrire un livre qu'on pourrait relire cinquante fois dans l'espace d'une vie - parce qu'il y a chez nous des imbéciles qui lisent les yeux fermés. Déjà le titre lui-même est piège : L'État (condition) honteux. (21-03-79).

La condition honteuse est bien celle du lecteur: il consomme, yeux fermés, des simulacres de réalité, il se laisse conditionner par des mots réduits à l'état honteux de cadavre. L'œil ne doit pas (seulement) servir à lire des livres, mais à lire la vie - cette vie dont l'écrivain se dit le " guetteur » infatigable, dont il extrait une sorte de précipité appelé art.

Il a besoin d'en avertir ses lecteurs. Tous les « avertissements » que Sony place au seuil de ses romans ont pour fonction d'inciter le lecteur à faire l'autre moitié du chemin, à trouver sa place dans la parcelle de vérité romanesque, bref à devenir, comme il le dit, un «co-créateur ». Faute de quoi, la lecture manque son rendez-vous avec l'écriture. Et Sony est sévère pour les responsables de l'échec:

Il y en a qui disent : quels sont vos passe-temps favoris. Alors ils disent : la lecture. C'est écœurant d'entendre quelqu'un prendre la lecture pour un passe-temps. [...] Pourquoi nous mangeons les vaches, parce que les vaches ne pensent pas; sinon elles se seraient organisées, elles auraient changé leur condition de vache. Alors tu dis, c'est un passe-temps, c'est étrange. Alors j'avertis tous ceux qui croient qu'ils vont entrer dans mon livre pour passer le temps. Et j'avertis tous ceux qui s'imaginent que moi, pour écrire une page, je prends deux, trois jours.

L'avertissement est clair : l'écriture ne progresse pas au même rythme que la lecture : elles sont incommensurables. La première crée du temps mesuré en pages volées au plaisir, la seconde ne fait que passer le temps, que le tuer par plaisir.

Impossible, donc, de mener les deux de front, dans le même temps. Intempestif, l'écrivain refuse la rumination des vaches : «Je ne peux pas relire ce que j'ai écrit, je ne peux rêver deux fois. » Le rêve, la vie et l'écriture sont pour Sony des entités à peu près interchangeables, où se confondent le comparant et le comparé, où s'abolit jusqu'à leur distinction. Car tous les trois vont - marchent - ensemble et toujours devant, vers un avant non tracé à l'avance. Celui qui rêve, vit, écrit ne se retourne pas.

À cet égard, Sony est l'anti-Orphée. Son chemin est sans retour. Une fois franchi «le premier pas »- titre du premier texte envoyé au Seuil -, il continue, pas forcément droit devant, mais en explorant tous «les chemins tortueux de la fable. » Car il ne se fait pas d'illusion sur les lendemains qui chantent sur l'air de l'avenir. On comprend ainsi un poème de jeunesse : à l'amoureuse qui disait "l'avenir ou rien", le poète en herbe répond par une mise en question : « l'avenir ? Ce passé à venir ».

On comprend aussi que Sony, de façon très conséquente, refuse de reprendre ce qu'il a donné une fois pour toutes :

Parce que moi, quand j'ai fini d'écrire le livre, ce n'est plus mon livre, ça devient le livre des autres. Le mien, c'est celui que je suis en train d'écrire, c'est celui que je vais bientôt écrire.

Une fois écrit, le livre n'appartient plus à celui qui l'a écrit mais au lecteur, un lecteur d'ailleurs « qui ne voit pas nécessairement la même chose que l'auteur ".

Cette différence de point de vue n'introduit pas un défaut dans la communication littéraire, elle en est le ressort essentiel. En tant que communication différée, l'œuvre littéraire, en effet, repose sur un malentendu crucial. On retrouve donc ici, mais 
transposé et valorisé, ce différend éthique que nous avions relevé à propos du Progrès « qui n'avance pas ». Entre l'auteur et son lectorat, le différend est aussi esthétique.

$\mathrm{Au}$ fond, c'est celui-là même qui oppose l'art et la culture, comme le remarque le cinéaste Godard dans un aphorisme aussi impeccable qu'impitoyable: «Il y a de la culture qui est la règle, et il $\mathrm{y}$ a de l'exception qui est de l'art - Il est de la règle de vouloir la mort de l'exception.»

Toujours le lecteur voudra reconduire l'auteur à sa culture, voire au consensus culturel ; toujours l'auteur lui opposera une facette inédite, inclassable et, disons-le, exceptionnelle - remettant ainsi à chaque fois son œuvre en jeu et en mouvement: Work in progress.

Pour ne pas conclure, ce poème « Testament » sur cette page orpheline.

\section{NOTES}

1. Bel exemple en lui-même de cette désorganisation génétique, ce fragment de poème transcrit d'une page orpheline qui se trouve glissée, par on ne sait quelle "étourderie ", dans le cahier manuscrit intitulé «Ces hommes qui fatiguent les chiffres » (numéro d'inventaire : 37 bis). Voir la reproduction scannée en annexe 1.

2. Cette association, qui s'était créée à la faveur de la transition démocratique au début des années 1990 pour faire pièce à l'ancienne UNEAC inféodée au PCT, parti unique honni et déchu par la Conférence nationale souveraine, comprenait les écrivains de la phratrie comme Sylvain Bemba, Emmanuel Dongala, Sony Labou Tansi, mais accueillait aussi bien, dans un esprit convivial et cosmopolite, des chercheurs, universitaires ou intellectuels du Congo et au Congo.

3. Sony Labou Tansi, L'autre monde. Écrits inédits, éd. Revue Noire, coll. « Soleil », 1997.

4. Cet ami de Sony, sans doute son plus proche confident, est journaliste. À ce titre, dans son émission Panorama littéraire qu'il animait dans les années 1980 à la radio nationale d'alors, La Voix de la Révolution congolaise, il avait mené plusieurs entretiens capitaux avec son ami devenu célèbre. Il m'en a gracieusement confié les enregistrements sur cassettes audio, qui ont été par la suite archivées sur support numérique par les soins de Frédéric Lavignette, journaliste à France Culture. Des extraits significatifs ont été transcrits et publiés en revue sous le titre "Le métier d'écrivain selon Sony Labou Tansi » dans « Approche génétique des écrits littéraires africains ", dossier littéraire réalisé et Greta Rodriguez-Antoniotti, Études Littéraires Africaines, $n^{\circ} 15,2003$, p. 31-39.

5. C'est dans cette période extrêmement troublée que la maison de Sylvain Bemba, située en plein quartier Bacongo, a été la cible d'une agression particulièrement destructrice : incendiée et rasée jusqu'aux fondations, cette maison renfermait sans doute le fonds d'archives le plus important de la «phratrie » des écrivains congolais - Sylvain en étant l'inventeur et le documentaliste le plus autorisé. Il m'avait donné à lire et copier un texte autographe de Sony, «Brouillon de lettre à Sylvain Bemba »; publiée dans L'autre monde (op. cit., p. 30-33), c'est la seule rescapée de cette catastrophe patrimoniale.

6. Cette plasticienne et écrivaine congolaise, proche de Tchicaya U Tam'si et de Sony Labou Tansi, a souvent aidé à les illustrer, par ses peintures, écrits ou photographies (voir notamment celles de la chambre et du banc de la maison de SLT à Makélékélé dans L'Autre monde, op. cit, p. 102). Elle 
a opéré la première transcription de la conférence de SLT à Bayardelle (à paraître dans Mélanges en hommage à Mukala Kadima-Nzuji).

7. Grâce à l'aide du comédien congolais Roch Banzouzi, alors réfugié à Yaoundé.

8. Voir notre première version de "Éditer les inédits de Sony Labou Tansi », dans ELA 15, op. cit., annexe 5, p. 51-52.

9. La mise en œuvre de ce parti pris éditorial n'alla pas sans difficultés pratiques et déontologiques. Voir nos affres et débats d'apprentis généticiens dans «Cap SLT : extraits du journal de bord ", Rupture, ${ }^{\circ}$ 6, 2006, Karthala, p. 199-224.

10. Jennifer Mangeard, sous la direction de Pierre-Marc de Biasi, université Paris 7-Denis Diderot, master Lettres, Arts et Pensée Contemporaine, Semestre 2006.

11. Voir en annexe 4 l'inventaire récapitulatif du fonds SLT qui s'est trouvé, du fait de la guerre civile de 1997, scindé en deux parties, l'une à Brazzaville et l'autre en France. Celle-ci a été exposée à la soirée du Louvre du 1 décembre 2011, puis déposée à l'ITEM (rue Pouchet), avant d'être enfin remise à la famille le 12 juillet pour dépôt conservatoire à la BFM de Limoges.

12. Lors de la mission suivante, en 2011 , je pourrais déplorer la disparition de deux tapuscrits d'importance (avec corrections manuscrites), Les Yeux du volcan et Une chouette petite vie bien osée, respectivement $\mathrm{n}^{\circ} 1$ et 2 de l'inventaire de 1996.

13. Voir en annexe 5 un exemple particulièrement flagrant de l'état de dégradation avancée de certains manuscrits.

14. 4168 exactement, si tant est que mon compte soit exact.

15. Occasion de remercier ici Suzanne Nzouzi, doctorante sur l'œuvre de SLT et collaboratrice assidue de l'équipe MF, qui a pris soin d'opérer une vérification systématique de la lisibilité du texte manuscrit sur chacune des numérisations. Son bilan s'établit à 36 scans où le texte a été coupé et qui devront être repris.

16. Dont l'un, successivement titré La France qui rend fou et La planète des Cignes (sic), a même fait la une du Point du samedi 11 octobre 2008, avec son éditorial intitulé «Sonynédit » et signé Patrick Besson.

17. Voir « Préface à la Planète des Signes ", dans L'Autre monde, op. cit., p. 34-37.

18. Voir la liste des archives sur le site de la Bibliothèque Francophone Multimédia, dont la numérisation n'est malheureusement pas encore disponible.

19. En 1979, avec un « Envoi » d'Henri Lopes.

20. Sur cette métaphore, voir mon « Le souffle et le travail », ELA, 15, p. 23-30.

21. Cette citation, ainsi que celles qui suivent, sont tirées des archives manuscrites ou audio inédites.

22. Voir tout de même : «Le métier d'écrivain selon Sony Labou Tansi, ELA, op. cit., p. 31-39.

23. Cf supra, note 16 .

\section{RÉSUMÉS}

Cet état des lieux des archives laissées par Sony Labou Tansi comprend deux volets. Le premier se présente comme un historique de la sauvegarde et de la valorisation des écrits qui constituent un continent immense et inédit. Leur histoire mouvementée est racontée à partir du moment où l'écriture s'arrête, lors la disparition de l'écrivain en 1995. Matériellement, les écrits sont couchés, pour la plupart, dans des cahiers d'écolier jetés en vrac au fond de la petite bibliothèque 
de sa maison de Makélékélé à Brazzaville. Sans date, parfois sans titre, ils représentent un véritable défi à la critique génétique qui cherche à les classer, les inventorier, les numériser et enfin à les éditer. Reconstituer leur parcours, au fil des multiples versions et transformations successives, aboutit à dresser la cartographie du labyrinthe textuel constitué non seulement du fonds SLT séparé entre la France et le Congo mais aussi des cahiers et lettres disséminés par l'auteur auprès de ses nombreux amis lecteurs et éditeurs. Dans le second volet, on s'attache à retrouver le fil conducteur de l'écriture, à en restituer la logique vitaliste et hypertextuelle. Si SLT écrivait comme on respire, son écriture, d'allure improvisée, décrite par la génétique comme « à processus ", relève, du point de vue anthropologique, du prophétisme scripturaire : un direécrire en progression continue - contre le progrès, sans jamais se retourner vers la lecture.

This appraisal of archives left by Sony Labou Tansi consists of two parts. The first one appears as a history of maintenance and of promotion of Tansi's writings, which are an immense and unknown land. Their eventful history starts, where the literary production ends: upon the death of Sony Labou Tansi in 1995. Physically, the writings are put down in schoolchild notebooks, thrown as they came at the bottom of a little library at the author's house of Makélékélé in Brazzaville. With no date, sometimes with no title on them, these notebooks appear as a real challenge for genetic critic, who is trying to class them, to make up their stocklist, to scan and to publish them. Reconstructing their history through multiple versions and successive transformations ends up with tracing a map of a textual labyrinth consisting not only of literary production of Sony Labou Tansi, broken up between France and the Congo, but also on notebooks and letters given by the author to the crowd of his friend readers and his publishers. In the second part of the article, we set out to retrace the creative process, to reconstruct the vitalist and hypertextual logic of Tansi's writing. If Sony Labou Tansi used to write as you breath, his writing with its improvised aspect, described by the genetic criticism as "à processus ", comes, from anthropological point of view, close to the scriptural prediction: a speech-writing in continuous progression - against the progress; never turning back towards reading.

\section{AUTEUR}

\section{NICOLAS MARTIN-GRANEL}

Chercheur-associé, ITEM 